\title{
Zinc Sulphide Ore Formation - a Cathodoluminescence and X-ray Examination
}

\section{Colin MacRae, Weihua Liu, Nick Wilson, Matthew Glenn and Aaron Torpy}

CSIRO Mineral Resources, Clayton, Victoria, Australia

To understand the ore formation process of hydrothermal zinc deposits we have grown zinc sulphide (ZnS) in the laboratory using a range of conditions to determine the optimum environment for crystal growth. The starting material is natural calcite which is introduced into a solution and under hydrothermal conditions dissolved and reprecipitated as anhydrite $\left(\mathrm{CaSO}_{4}\right)$ together with $\mathrm{ZnS}$. Initial experiments show that the $\mathrm{ZnS}$ nucleates on the surface of the anhydrite and forms crystal bundles (rosettes) which accumulate into layers of tens of microns, Fig 1. When examined using an electron microprobe equipped with a fixed grating spectrometer to detect cathodoluminescence $(\mathrm{CL})^{1}$ the outer growth areas are bright while the inner large grains showed weaker CL, Fig 2. The centre responsible for the blue-green emission in $\mathrm{ZnS}$ is a self-activated luminescent centre ${ }^{2}$ and is most likely associated with chlorine impurites ${ }^{3}$.The presence of chlorine was confirmed by EDS and elevated chlorine levels were found in the ZnS-bulk compared to the $\mathrm{ZnS}$-nano. The chlorine impurities substitute for the $\mathrm{S}$ atoms and form with $\mathrm{Zn}$ vacancy acceptor complex defects ${ }^{2}$. It is likely that the $\mathrm{ZnS}$ centres in the nano size crystals are enhancing the emission compared to the bulk and possibly more centres can emit in the nano crystals.

The anhydrite crystals formed under the hydrothermal conditions were CL active and showed a number of rare earth (RE) emission centres including ${ }^{4} \mathrm{Ce}^{3+}, \mathrm{Dy}^{3+}, \mathrm{Er}^{3+}, \mathrm{Sm}^{3+}$, and $\mathrm{Nd}^{3+}$. A broad peak observed in the anhydrite spectrum, centred at $550 \mathrm{~nm}$, was attributed to $\mathrm{Mn}^{2+}$. The source of the RE ions was from the calcite $\left(\mathrm{CaCO}_{3}\right)$ as all other materials were analytical grade reagents. Subtle weak zoning was observed in the anhydrite and its roles as a nucleation site for the $\mathrm{ZnS}$ was investigated further. To improve the light sensitivity and the wavelength resolution a second spectrometer equipped with a movable grating was attached to the EPMA (Princeton instruments ACTON spectrometer, model \#2300i, 300 1/mm grating, with a PIXIS $100 \mathrm{CCD}$ camera operated at $\left.-60^{\circ} \mathrm{C}\right)$. The mapping conditions employed were beam conditions of $12 \mathrm{kV}, 40 \mathrm{nA}$, dwell time of $30 \mathrm{~ms}$ and step size of $400 \mathrm{~nm}$ which were the same as those used for the fixed grating spectrometer. The enhanced sensitivity of the Princeton spectrometer and CCD revealed subtle banding in the anhydrite not previously observed. The peak FWHM of the RE ions did not significantly change with the higher resolution spectrometer indicating either thermal broadening, poor crystallinity or substitutions into multiple sites. Imaging at liquid nitrogen will be used in the future to investigate whether the RE peaks were thermal broadening.

Further studies of the $\mathrm{ZnS}$ growth will focus on doping with gallium, indium, germanium and thallium. This is part of a wider valuable elements study. These single element doped $\mathrm{ZnS}$ samples will provide excellent reference spectra for the use of standards for both cathodoluminescence and potentially as secondary standards for microanalysis. 


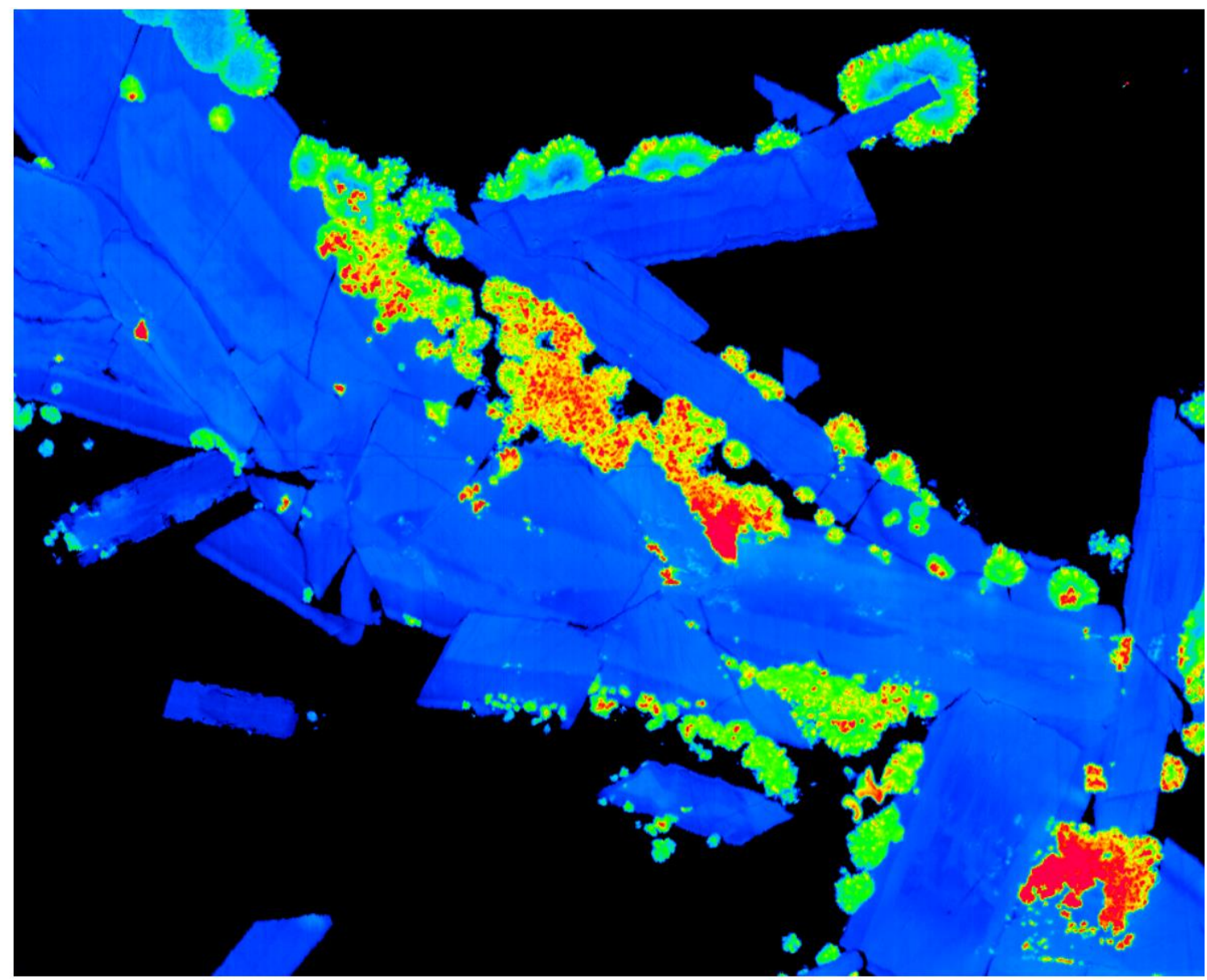

$100 \mu \mathrm{m}$

Figure 1. Cathodoluminescence panchromatic image showing zinc sulphide crystals (red-green) nucleating on a calcium sulphate crystal (light blue-blue). The $\mathrm{ZnS}$ emission is enhanced by the presence of chlorine substitution.

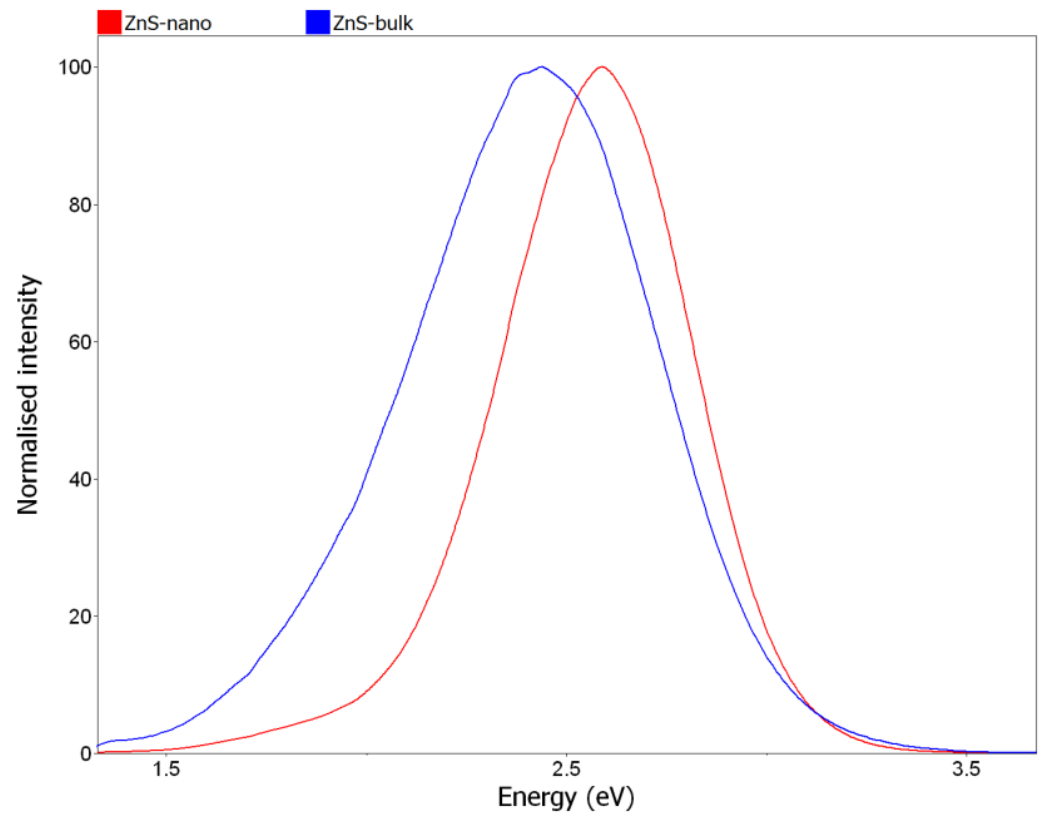


Figure 2. Cathodoluminescence spectra form freshly nucleated nano zinc sulphide crystals versus bulk zinc sulphide. The emission is due to a self-activated luminescent centre and is most likely associated with chlorine impurites.

\section{References}

1. MacRae et al. Mineralogy and Petrology. 107, 3 (2013), pp 429-440

2. Hichou et al. Semiconductor Science and Technology 19 (2004) pp 230-235

3. Yamaga et al. Crystal Growth. 106 (1990) pp 683-689

4. Baumer et al. Chemical Geology. 138 (1997), pp 73-80 